CLINICAL STUDY

\title{
Hypocortisolemic clamp unmasks jointly feedforward- and feedback-dependent control of overnight ACTH secretion
}

\author{
Ali Iranmanesh and Johannes D Veldhuis ${ }^{1}$ \\ Endocrine Section, Department of Medicine, Salem Veterans Affairs Medical Center, Salem, Virginia 24153, USA and ${ }^{1}$ Endocrine Research Unit, \\ Department of Internal Medicine, Mayo School of Graduate Medical Education, Clinical Translational Science Center, Mayo Clinic, Rochester, Minnesota \\ 55905, USA \\ (Correspondence should be addressed to J D Veldhuis; Email: veldhuis.johannes@mayo.edu)
}

\begin{abstract}
Background: ACTH secretion is under hypothalamic stimulatory (feedforward) and adrenal inhibitory (feedback) control.

Hypothesis: Assessment of overnight ACTH secretion during a hypocortisolemic clamp will permit the estimation of changing feedforward and feedback.

Subjects: Seven healthy men.

Interventions: An oral dose of placebo (PLAC), metyrapone (METY, $3 \mathrm{~g}$ ), or ketoconazole (KTCZ, $1.2 \mathrm{~g}$ ) was given at midnight $(\mathrm{MN})$ to block glucocorticoid synthesis. Plasma ACTH was sampled every $10 \mathrm{~min}$ (MN to $0800 \mathrm{~h}$ ).

Analysis: Variable-waveform deconvolution analysis of ACTH secretion and approximate entropy (ApEn) analysis of pattern regularity.

Results: Compared with PLAC, administration of METY and KTCZ reduced morning cortisol concentrations by $\geq 77$ and $54 \%$ respectively $(P<0.001)$. Hypocortisolemia elevated pulsatile ACTH secretion by 8.2- (METY) and 5.3-fold (KTCZ; both $P<0.001$ ). Basal ACTH secretion rose by 3.4-fold under METY-induced cortisol depletion $(P=0.020)$. ACTH secretory-burst shape and half-life were stable. ApEn of ACTH release declined overnight $(P=0.021)$ and with the drug $(P=0.001)$, denoting enhanced feedforward coordination.

Conclusion: The combined data predict overnight amplification and coordination of hypothalamic feedforward drive onto ACTH release. Therefore, disruption of either mechanism might contribute to clinical pathophysiology, such as late-day elevations of cortisol output in fasting, alcoholism, depression, or aging.
\end{abstract}

European Journal of Endocrinology 159 561-568

\section{Introduction}

Adrenocorticotrophin (ACTH) is secreted in a pulsatile, nycthemeral, and entropic (feedback-sensitive) fashion (1-4). The fundamental physiological issue emerges of how all three of pulsatile, nycthemeral, and feedback modes of ACTH secretion are interlinked. Discrete pulses are generated by episodic hypothalamic release of corticotropin-releasing hormone (CRH) and arginine vasopressin (AVP), which evoke ACTH secretory bursts under feedback repression by glucocorticoids (5-11). Analysis of pulsatile ACTH secretion thus provides a window into hypothalamo-pituitary control mechanisms (4). Nycthemeral rhythmicity is endowed by 24-h variations in secretory burst size and/or number (12). However, the manner in which feedforward (hypothalamic stimulation) and feedback (glucocorticoid inhibition) coordinate pulsatile and nycthemeral ACTH secretion is not known. Feedback-dependent effects can be quantified objectively with a regularity (orderliness) statistic, approximate entropy (ApEn) (13-15).
More regular (less entropic) secretory patterns signify greater feedback coordination in both mathematical and empirical models $(16,17)$.

One strategy to examine interlinked mechanisms of ACTH control would be to assess feedback-regulated pulsatile secretion overnight when the transition from nadir-to-zenith hormone output normally occurs. The premise advanced is that feedback decreases and feedforward increases during the nighttime, thereby amplifying pulsatile ACTH secretion in the morning. Pulsatile secretion can be quantified by deconvolution analysis and feedback-dependent regularity by the ApEn statistic $(14,15,18,19)$. Deconvolution analysis yields objective estimates of basal and burst-like secretion, whereas ApEn confers insights into negative feedback independently of pulsatility changes. Under this imprimatur, we hypothesized that overnight augmentation of pulsatile ACTH secretion requires both attenuation of negative feedback and amplification of feedforward. 


\section{Methods}

\section{Subjects and protocol}

Healthy unmedicated men ( $N=6$ placebo (PLAC), $N=7$ for both drugs, range of age 35-52 years and body mass index $24-33 \mathrm{~kg} / \mathrm{m}^{2}$ ) participated after providing written informed consent approved by the institutional review board of the Salem-Roanoke Veterans Affairs Medical Center. Each subject had an unremarkable medical history and physical examination, and normal screening tests of hepatic, renal, hematological, and endocrine thyroid-stimulating hormone (TSH, morning cortisol, insulin-like growth factor-I and testosterone) function. Volunteers underwent randomly ordered overnight sampling studies at least 2 weeks apart. Sessions entailed placement of a forearm i.v. catheter at $2200 \mathrm{~h}$, and oral administration of PLAC, (METY $3 \mathrm{~g}$ ), or ketoconazole (KTCZ $1.2 \mathrm{~g}$ ) with a snack at midnight (MN). Both drugs lower cortisol concentrations, albeit via different sites of steroidogenic blockade, viz., CYP11B and CYP11A
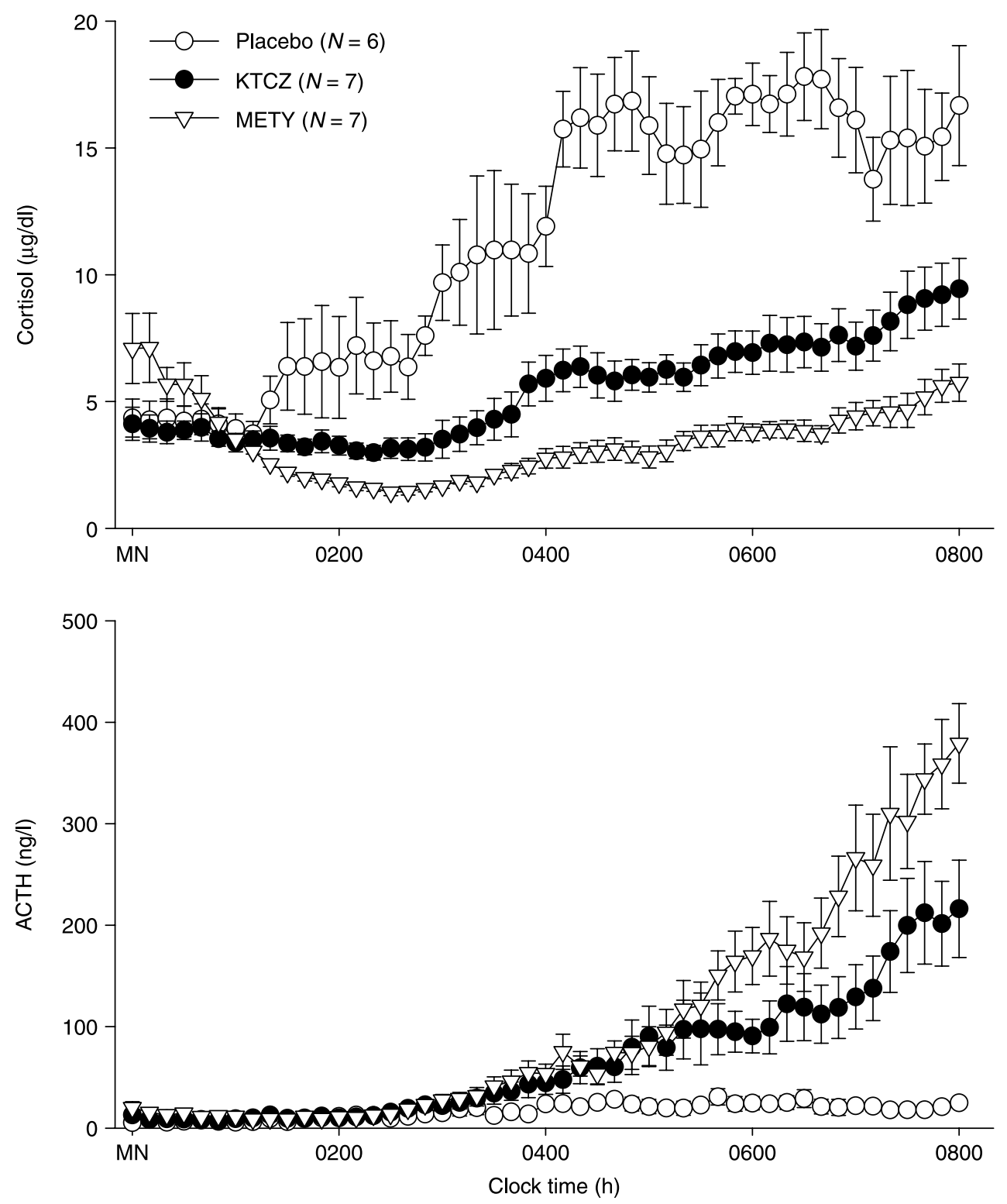

Figure 1 Time courses of plasma cortisol (top) and ACTH (bottom) concentrations in six healthy men sampled every $10 \mathrm{~min}$ from midnight (MN) to $0800 \mathrm{~h}$. Each subject received oral PLAC, KTCZ, or METY at MN, as indicated. Data are the mean \pm S.E.M. 
respectively $(9,20)$. Thus, their use is complementary to verify that hypocortisolemia rather than drug type drives ACTH changes. Plasma was withdrawn every $10 \mathrm{~min}$ from $\mathrm{MN}$ to $0800 \mathrm{~h}$ in chilled EGTA-containing plastic tubes on ice and centrifuged immediately in the cold before freezing at $-70{ }^{\circ} \mathrm{C}$ for later assay of ACTH and cortisol.

\section{Hormone assays}

ACTH and cortisol concentrations were measured by immunoradiometric and solid-phase RIA respectively, as described earlier $(10,11)$. Sensitivity was $2 \mathrm{ng} / \mathrm{l}$ for ACTH and $2 \mu \mathrm{g} / \mathrm{dl}$ ( $58 \mathrm{nmol} / \mathrm{l}$ ) for cortisol. All samples from each subject were assayed in batch. Intraassay coefficient of variation (CV) values for ACTH and cortisol were 6.5 and $5.8 \%$ and interassay $C V$ values 8.5 and $6.9 \%$ respectively.

\section{Deconvolution analysis}

Overnight ACTH concentration time series (total $8 \mathrm{~h}$ ) were analyzed by way of a recently developed variablewaveform deconvolution method (19). The automated Matlab program first detrends and normalizes concentrations to the unit interval $[0,1]$ (18). Second, successive potential pulse-time sets are created by an incremental smoothing process (a nonlinear adaptation of the heat-diffusion equation), which deletes the least significant nadir one at a time. Third, maximumlikelihood expectation parameter estimation is used to calculate secretion and elimination rates simultaneously for each candidate pulse-time set. The model specifies basal secretion $\left(\beta_{0}\right)$, a slow-phase halflife $\left(\alpha_{2}\right)$, secretory-burst mass $\left(\eta_{0}, \eta_{1}\right)$, random effects on burst mass $\left(\sigma_{\mathrm{A}}\right)$, procedural/measurement error $\left(\sigma_{\varepsilon}\right)$, and a three-parameter flexible Gamma probability distribution to embody secretory-burst waveform $\left(\beta_{1}\right.$, $\left.\beta_{2}, \beta_{3}\right)$. The rapid phase half-life of ACTH was assumed to be $3.5 \mathrm{~min}$ representing $37 \%$ of total decay. And, fourth, the Akaike information criterion is applied to distinguish objectively among candidate pulse-time sets (21). Observed interpulse intervals are described by a two-parameter Weibull renewal process (more general form of a Poisson process). Units of parameters are burst frequency (number per $24 \mathrm{~h}, \lambda$ of Weibull distribution), regularity of interpulse intervals (unitless $\gamma$ of Weibull), slow half-lives (min), basal and pulsatile secretion rates (concentration units/24h), mass secreted per burst (concentration units), and waveform mode (time delay to maximal secretion after burst onset, min) $(18,19)$.

\section{ApEn analysis}

Approximate entropy, ApEn (1, 75\%), was used as a scale- and model-independent regularity statistic to quantify the orderliness of ACTH release in each 2-h block (13-15, 22). Higher ApEn denotes greater disorderliness of the secretion process, and conversely for low ApEn. Mathematical models and clinical experiments establish that greater pattern regularity signifies heightened feedback control with high sensitivity and specificity (both $>90 \%)(16,23,24)$.

\section{Statistical analysis}

Two-way ANOVA in a $4 \times 3$ factor repeated-measures design was used to assess the individual and combined (interactive) impact of 2-h time segments (four factors) and drug treatment (three factors) on ACTH and cortisol concentrations, ACTH secretory parameters, and ACTH ApEn. $P<0.05$ was construed as significant. Data are expressed as the mean \pm s.e.m. The significance of any set of six or seven slopes was tested by the chisquare statistic applied to -2 times the sum of the natural logarithms of the $P$ values at 1 degree of freedom (25).
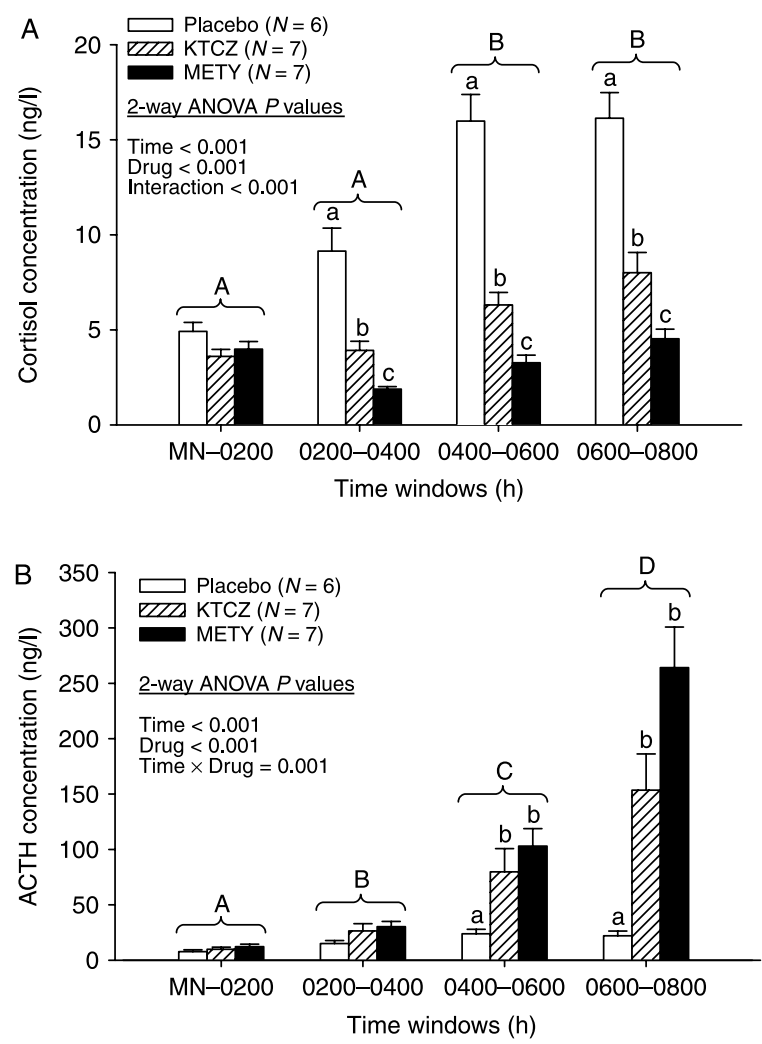

Figure 2 Mean cortisol $(A)$ and $\mathrm{ACTH}(B)$ concentrations monitored overnight. Data were segmented into 2- $h$ windows and subjected to two-way ANOVA in a repeated-measures design. Different alphabetic letters denote significant contrasts among time segments independently of treatment (capital letters) and among PLAC, KTCZ, and METY treatments within any given time segment (lower-case letters). Data are the mean \pm s.E.M. $(N=6$ subjects). 

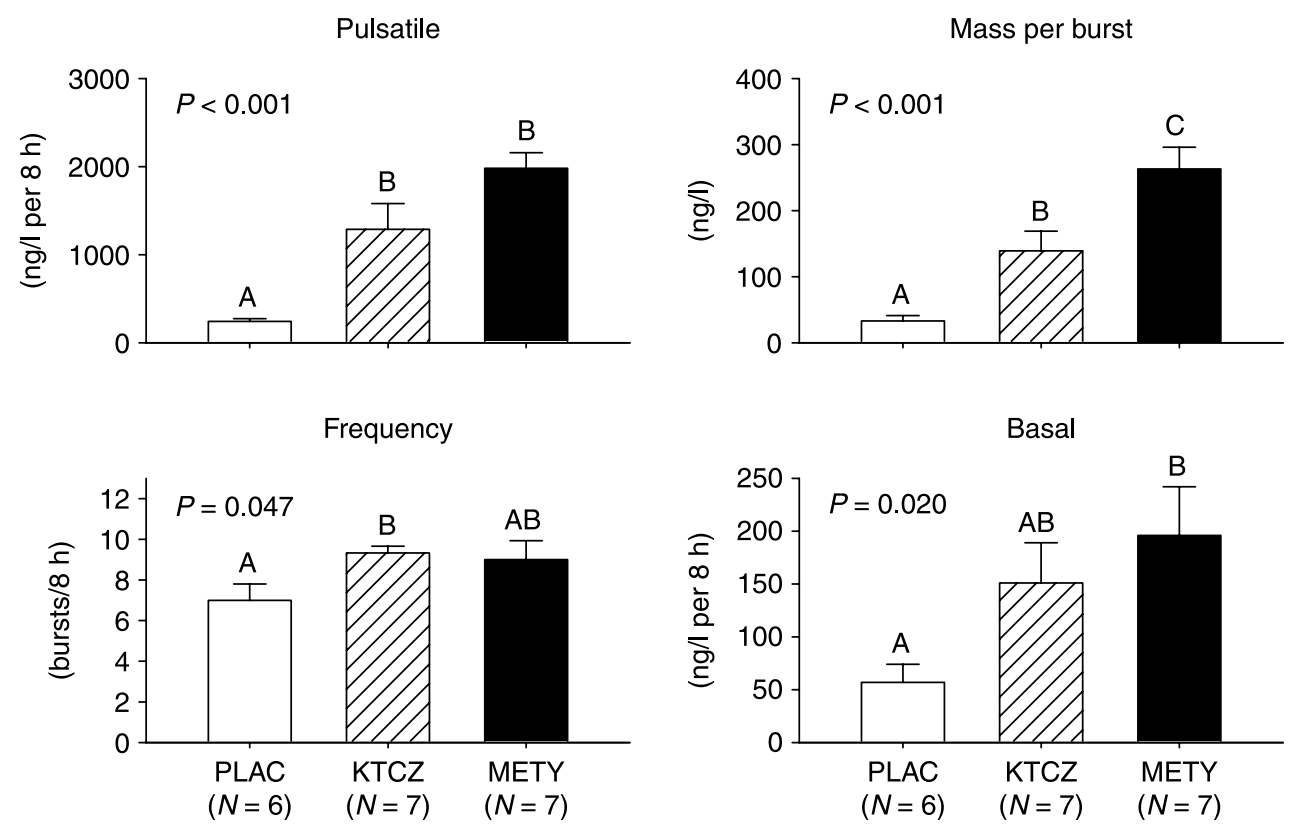

Figure 3 Nocturnal pulsatile ACTH secretion, ACTH secretory-burst mass, ACTH pulse frequency, and basal ACTH secretion. Data are 8-h estimates (mean \pm S.E.M., $N=6$ ). Different superscripts differ significantly by one-way ANOVA and Tukey's post hoc test.

\section{Results}

Cohort mean cortisol and ACTH concentration time series are depicted in Fig. 1. Visual inspection indicated that both steroidogenic inhibitors were effective in suppressing cortisol and elevating ACTH concentrations. In the PLAC session, ACTH concentrations rose by 2.8 -fold and cortisol concentrations by 3.5 -fold at $0600-0800 \mathrm{~h}$ compared with corresponding ACTH and cortisol values measured in the same subjects at MN-0200 (Fig. 2A and B). Administration of METY and KTCZ reduced cortisol concentrations by $45-60 \%(\mathrm{MN}-0400 \mathrm{~h})$ and $54-77 \%$ (0400-0800 h) compared with PLAC responses evaluated at the same times $(P<0.001$ treatment effect and $P<0.001$ time effect by two-way ANOVA; Fig. 2A). ACTH responses were evaluated in relation to PLAC and drug effects in three ways. First, METY-induced hypocortisolemia increased mean ACTH concentrations during the successive 2-h intervals MN-0200, 0200-0400, 0400-0600, and 0600-0800 h by 1.5-, 2-, 4-, and 12-fold respectively, compared with time-matched effects of PLAC. Analogous KTCZ effects were 1.3-, 1.7-, 3.3-, and 7.0-fold compared with time-matched PLAC $(P<0.001$ for both drug and time effects and $P<0.001$ for drug $\times$ time interaction; Fig. 2B). The data document significant and comparable disinhibition of negative feedback after $0400 \mathrm{~h}$ by both steroidogenic inhibitors. Second, exposure to METY and KTCZ augmented mean 0600-0800 h ACTH concentrations by 34- and 20-fold respectively, compared with exposure to PLAC during the early-night interval of MN-0200 h $(P<0.001)$. Third, METY and KTCZ elevated mean ACTH concentrations at 0600-0800 h by 22- and 25-fold over those measured at
MN-0200 h under the same drug treatment. Thus, ACTH feedforward during overnight hypocortisolemia increases multifold principally between $0400 \mathrm{~h}$ and $0800 \mathrm{~h}$.

Deconvolution analysis of each 8-h time series established that elevated morning ACTH concentrations result primarily from greater pulsatile rather than basal ACTH release $(P<0.001$; Fig. 3$)$. In particular, mean pulsatile ACTH secretion over the 8-h session increased markedly and similarly after administration of METY (8.3-fold) and KTCZ (5.3-fold) compared with PLAC (both contrasts $P<0.001$ ). Augmented pulsatile secretion in turn was due to both an 8.0- (METY) and 4.2-fold (KTCZ) increase in ACTH secretory-burst mass $(P<0.001$ for both and $P<0.01$ for their difference). There was a lesser 1.33-fold stimulatory effect of KTCZ on ACTH secretory-burst frequency $(P=0.047)$ and a nonsignificant 1.28-fold effect of METY $(P=0.11)$. Basal ACTH secretion was $5.7 \pm 17 \mathrm{ng} / \mathrm{l}$ per $8 \mathrm{~h}$, and rose by 3.4-fold during METY administration $(P=0.020)$ and 2.7 -fold during KTCZ administration $(P=0.052)$. The foregoing responses were selective, because ACTH half-life, secretory-burst

Table 1 Other parameters of adrenocorticotrophin dynamics.

\begin{tabular}{lccc}
\hline Parameter & $\begin{array}{c}\text { PLAC } \\
(N=6)\end{array}$ & $\begin{array}{l}\text { KTCZ } \\
(N=7)\end{array}$ & $\begin{array}{c}\text { METY } \\
(N=7)\end{array}$ \\
\hline Half-life $(\mathrm{min})$ & $21 \pm 1.1$ & $22 \pm 0.80$ & $22 \pm 0.70$ \\
$\begin{array}{l}\text { Mode of secretory } \\
\quad \text { burst }(\mathrm{min})\end{array}$ & $16 \pm 1.7$ & $17 \pm 1.6$ & $17 \pm 1.6$ \\
$\quad$ Variability of pulsing $(\gamma)$ & $4.3 \pm 1.8$ & $2.6 \pm 0.36$ & $2.9 \pm 0.65$
\end{tabular}

Data are the mean \pm S.E.M. 

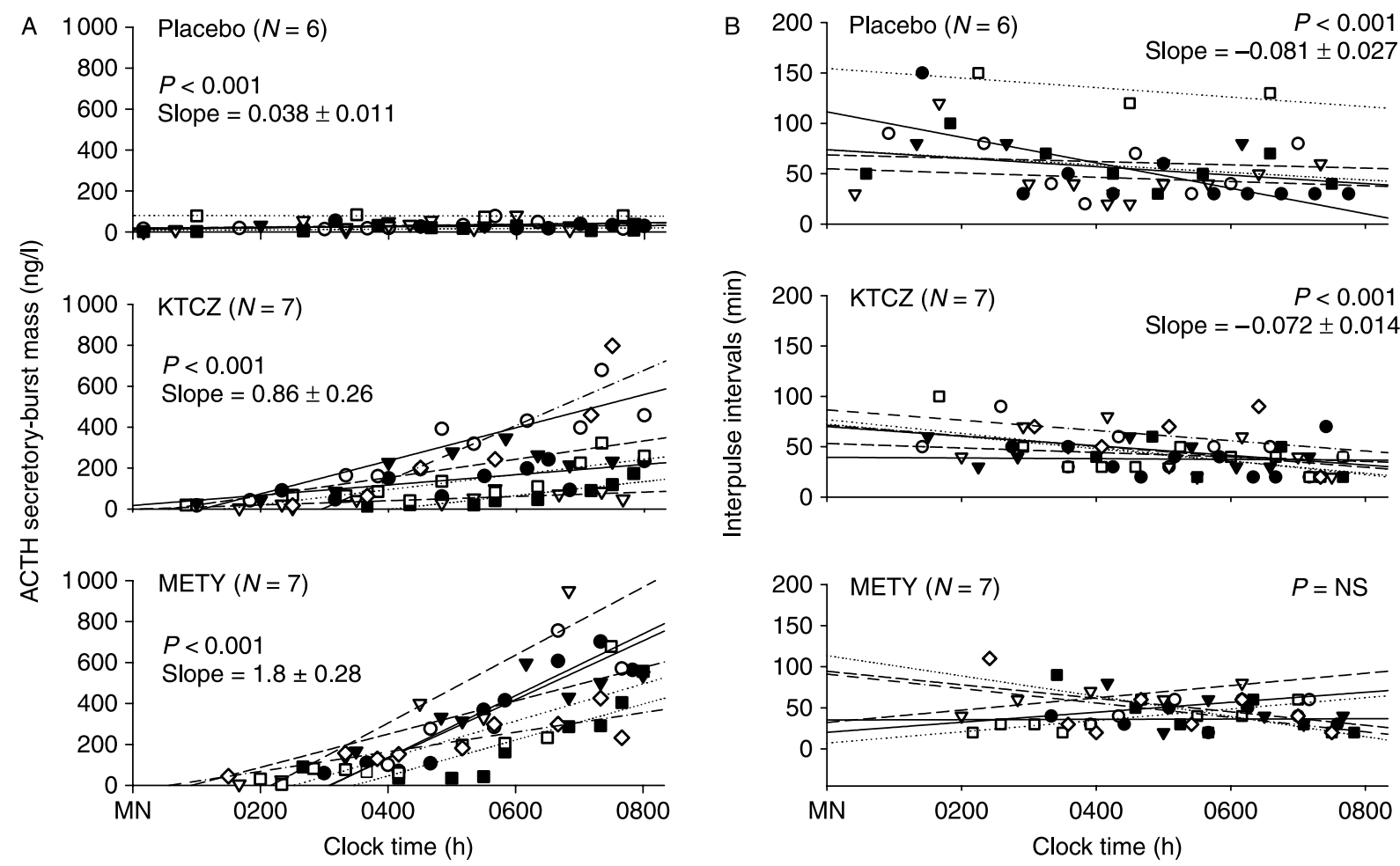

Figure 4 Linear regression of ACTH secretory-burst mass $(A)$ and interpulse intervals $(B)$ on time in six men exposed to placebo, KTCZ, and METY, as marked. Each symbol type denotes a different subject. Significance is defined by the aggregate $P$ value (Methods).

shape and interpulse-interval variability did not change (Table 1). The time dependence of augmented ACTH secretory-burst size was highly significant after each of PLAC, KTCZ, and METY administration (all $P<0.001$; Fig. 4A). Conversely, overnight ACTH interburst-interval lengths declined significantly $(P<0.001)$ during PLAC and KTCZ but not METY administration (Fig. 4B).

Postulated changes in feedback onto ACTH were assessed by ApEn analysis. Lower ApEn denotes greater feedback coordination (Methods). Two-way ANOVA revealed a prominent overnight decline in ACTH ApEn $(P=0.021$ a drug-specific effect $P=0.001)$, and no interaction between time and drug $(P=0.14$; Fig. 5A). The most prominent change occurred in the METY session, indicating that a normal overnight rise in plasma cortisol concentrations is not required to mediate the ApEn decline. Greater regularity (lower ApEn) was due to more reproducible ACTH secretoryburst mass sequences $(P=0.033)$ rather than to more regular interpulse intervals (Fig. 5B).

\section{Discussion}

The present investigation combined deconvolution analysis and ApEn estimates with a hypocortisolemic clamp to appraise the mechanisms that regulate ACTH secretion across the nighttime transition from nadir to zenith cortisol production in healthy men. The rationale for using two pharmacologic agents is that either could have a nonspecific effect, but it is unlikely that both would have the same nonspecific effect. Congruity of outcomes with structurally distinct inhibitors provides strong corroboration of the role of hypocortisolemia per se. The collective data indicate that i) glucocorticoid negative feedback on pulsatile ACTH secretion normally increases overnight after $0200 \mathrm{~h}$; ii) hypothalamic feedforward increases after $0400 \mathrm{~h}$ and more markedly than feedback; and iii) time of night and hypocortisolemia together determine coordinated hypothalamic drive of ACTH secretion.

A significant unexpected finding was that ApEn declined overnight with the lowest values reached by 0400-0600 $\mathrm{h}$ in the METY group, denoting maximal feedforward coordination at this time. The decline in ApEn was intermediate for KTCZ. Lower ApEn in biological and mathematical ensemble systems signifies greater negative feedback and/or more coordinated feedforward inputs $(16,17)$. The fact that ACTH ApEn declined when cortisol concentrations fell under druginduced feedback withdrawal indicates that cortisol concentrations are not the sole determinant of feedforward-dependent ACTH secretory regularity. One plausible organizing signal is somatostatin, which inhibits CRH and to a lesser degree AVPs stimulation of ACTH release (26-28). The role of AVP is putatively 

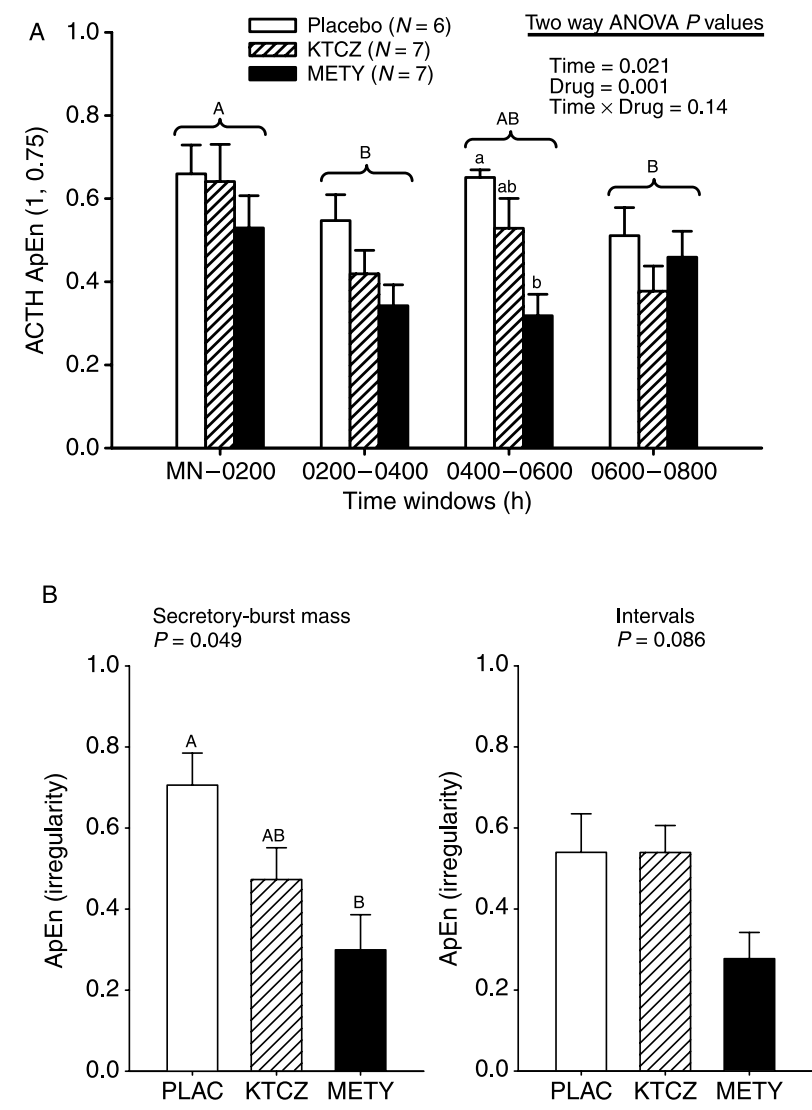

Figure 5 (A) Overnight evolution of ACTH ApEn, an ensemble regularity statistic. Lower ApEn denotes more orderly secretory patterns. (B) Irregularity (ApEn) of 8-h sequences of ACTH secretory-burst mass (left) and interpulse-interval length (right). $P$ values reflect results of ANOVA. Data are presented otherwise as described in Fig. 2.

most evident in stress. Greater hypothalamic somatostatin outflow may occur following deep sleep, since growth hormone $(\mathrm{GH})$ responses to a fixed dose of GHRH are then inhibited (29). In sum, ACTH regularity enhancement detected by ApEn might reflect enhanced coordination between stimulation by CRH (and AVP) and inhibition by somatostatin $(30,31)$. Interpulse secretion of somatostatin, a peptide that selectively blocks secretory-vesicle release, would favor corticotrope accumulation of exocytotic granules for discharge before the next CRH (or AVP) pulse. This concept could explain the concomitant increase in ACTH secretoryburst mass and decrease in ApEn observed between $0400 \mathrm{~h}$ and $0800 \mathrm{~h}$.

Hypocortisolemia selectively augmented nighttime ACTH secretory-burst mass (by 5.1- to 7.1-fold) and in lesser measure frequency (by 1.3-fold). By contrast, ACTH pulse-time regularity, ACTH half-life, and ACTH secretory-burst shape were not affected by hypocortisolemia. A larger 24-h study also found no evident regulation of ACTH pulsing regularity (11). Exploratory regression analysis revealed no consistent effect of body-mass index on any secretory measures, except for an unexplained negative correlation between basal ACTH secretion and body mass index in the KTCZ group only.

Few studies have evaluated ACTH secretion across the nighttime cortisol nadir. The present analyses show that reduction of cortisol concentrations by adrenal steroidogenic blockade with METY between 0200 and $0400 \mathrm{~h}$ stimulates ACTH secretion by 2.0-fold compared with PLAC administered at the same time. Thus, diurnally low cortisol concentrations continue to repress ACTH secretion even when the corticotropic axis is minimally active. Hypocortisolemia imposed by METY during the interval 0600-0800 h amplified mean ACTH concentrations by 34-fold with respect to the PLAC-associated nadir ( $\mathrm{MN}-0200 \mathrm{~h})$, 12-fold over the contemporaneous (0600-0800 h) PLAC control, and 22-fold over the METY nadir (MN-0200 h). We postulate that the 34-fold increase across the nighttime reflects both feedback disinhibition and strong feedforward drive. In this model, 12-fold disinhibition of ACTH output over the nighttime-matched PLAC response indicates the existence of significant negative feedback after the MN cortisol nadir, whereas 22-fold augmentation of ACTH secretion over the MN low-cortisol milieu reflects prominent feedforward at 0600-0800 h. These data are congruent with the clinical principles of minimizing ACTH suppression by administering synthetic glucocorticoids once daily at midnight and testing feedback escape over the same interval.

Little is known about the regulation of basal (nonpulsatile) ACTH secretion. Available evidence suggests that constitutive peptide release may reflect a low frequency of partial emptying of secretory vesicles at the plasma membrane $(32,33)$. Deconvolution analysis predicted a 3.4-fold rise in basal ACTH secretion during METY compared with PLAC administration. The effect of KTCZ, which lowered cortisol less markedly, was less prominent, suggesting that reduced cortisol availability augments basal ACTH secretion.

In conclusion, experimentally imposing hypocortisolemia via structurally distinct drugs unmasks (a) cortisol feedback-dependent regulation of ACTH secretory-burst mass and (b) time of night-dependent regulation of both ACTH secretory-burst mass and the orderliness of the ACTH secretion process in healthy men. These outcomes are consistent with a dynamic model in which both cortisol feedback and hypothalamic feedforward change overnight. If this model is valid, then corticotropic-axis pathophysiology such as late-day hypercortisolemia in fasting, alcoholism, depression and aging (34-37), might arise from disruption of nighttime regulatory mechanisms.

\section{Declaration of interest}

The authors declare that there is no conflict of interest that would prejudice the impartiality of this scientific work. 


\section{Funding}

Supported in part via the Clinical Translational Research-Center Grant Number UL 1 RR024150 to Mayo Clinic and Foundation from the National Center for Research Resources (Rockville, MD, USA), R2 1 AG29215 and R01 DK73148 from the National Institutes of Health (Bethesda, MD, USA).

\section{Acknowledgements}

We thank Kay Nevinger and Donna Scott for support of manuscript preparation; Ashley Bryant for graphical presentations; the Mayo Immunochemical Laboratory for assay assistance; and the Mayo research nursing staff for implementing the protocol.

\section{References}

1 Gallagher TF, Yoshida K, Roffwarg HD, Fukushida DK, Weitzman ED \& Hellman L. ACTH and cortisol secretory patterns in man. Journal of Clinical Endocrinology and Metabolism 197336 1058-1073.

2 Horrocks PM, Jones AF, Ratcliffe WA, Holder G, White A, Holder R, Ratcliffe JG \& London DR. Patterns of ACTH and cortisol pulsatility over twenty-four hours in normal males and females. Clinical Endocrinology 199032 127-134.

3 Iranmanesh A, Short D, Lizarralde G \& Veldhuis JD. Intensive venous sampling paradigms disclose high-frequency ACTH release episodes in normal men. Journal of Clinical Endocrinology and Metabolism 199071 1276-1283.

4 Keenan DM, Licinio J \& Veldhuis JD. A feedback-controlled ensemble model of the stress-responsive hypothalamo-pituitaryadrenal axis. PNAS 200198 4028-4033.

5 Rivier C \& Vale W. Modulation of stress-induced ACTH release by corticotropin-releasing factor, catecholamines and vasopressin. Nature 1983305 325-327.

6 Fink G, Robinson IC \& Tannahill LA. Effects of adrenalectomy and glucocorticoids on the peptides CRF-41, AVP and oxytocin in rat hypophysial portal blood. Journal of Physiology 1988401 329-345.

7 Fehm HL, Voigt KH, Kummer G, Lang R \& Pfeiffer EF. Differential and integral corticosteroid feedback effects on ACTH secretion in hypoadrenocorticism. Journal of Clinical Investigation 197963 $247-253$.

8 Erkut ZA, Pool C \& Swaab DF. Glucocorticoids suppress corticotropin-releasing hormone and vasopressin expression in human hypothalamic neurons. Journal of Clinical Endocrinology and Metabolism 199883 2066-2073.

9 Veldhuis JD, Iranmanesh A, Naftolowitz D, Tatham N, Cassidy F \& Carroll BJ. Corticotropin secretory dynamics in humans under low glucocorticoid feedback. Journal of Clinical Endocrinology and Metabolism 200186 5554-5563.

10 Keenan DM, Roelfsema F \& Veldhuis JD. Endogenous ACTH concentration-dependent drive of pulsatile cortisol secretion in the human. American Journal of Physiology. Endocrinology and Metabolism 2004287 E652-E661.

11 Keenan DM \& Veldhuis JD. Cortisol feedback state governs adrenocorticotropin secretory-burst shape, frequency and mass in a dual-waveform construct: time-of-day dependent regulation. American Journal of Physiology 2003285 R950-R961.

12 Veldhuis JD, Iranmanesh A, Johnson ML \& Lizarralde G. Twentyfour hour rhythms in plasma concentrations of adenohypophyseal hormones are generated by distinct amplitude and/or frequency modulation of underlying pituitary secretory bursts. Journal of Clinical Endocrinology and Metabolism 199071 1616-1623.

13 Pincus SM. Irregularity and asynchrony in biologic network signals. Methods in Enzymology 2000321 149-182.

14 Hartman ML, Pincus SM, Johnson ML, Matthews DH, Faunt LM, Vance ML, Thorner MO \& Veldhuis JD. Enhanced basal and disorderly growth hormone secretion distinguish acromegalic from normal pulsatile growth hormone release. Journal of Clinical Investigation 199494 1277-1288.

15 Pincus SM, Gevers E, Robinson ICAF, van den Berg G, Roelfsema F, Hartman ML \& Veldhuis JD. Females secrete growth hormone with more process irregularity than males in both human and rat. American Journal of Physiology 1996270 E107-E115.

16 Veldhuis JD, Straume M, Iranmanesh A, Mulligan T, Jaffe CA, Barkan A, Johnson ML \& Pincus SM. Secretory process regularity monitors neuroendocrine feedback and feedforward signaling strength in humans. American Journal of Physiology 2001280 R721-R729.

17 Pincus SM. Quantifying complexity and regularity of neurobiological systems. Methods in Neuroscience 199528 336-363.

18 Keenan DM, Chattopadhyay S \& Veldhuis JD. Composite model of time-varying appearance and disappearance of neurohormone pulse signals in blood. Journal of Theoretical Biology 2005236 242-255.

19 Keenan DM, Roelfsema F, Biermasz N \& Veldhuis JD. Physiological control of pituitary hormone secretory-burst mass, frequency and waveform: a statistical formulation and analysis. American Journal of Physiology 2003285 R664-R673.

20 Labeur M, Arzt E, Stalla GK \& Paez-Pereda M. New perspectives in the treatment of Cushing's syndrome. Current Drug Targets. Immune, Endocrine and Metabolic Disorders 20044 335-342.

21 Akaike $\mathrm{H}$. A new look at the statistical model identification. IEEE Transactions on Automatic Control 197419 716-723.

22 Pincus SM, Hartman ML, Roelfsema F, Thorner MO \& Veldhuis JD. Hormone pulsatility discrimination via coarse and short time sampling. American Journal of Physiology 1999277 E948-E957.

23 Veldhuis JD, Metzger DL, Martha PM Jr, Mauras N, Kerrigan JR, Keenan B, Rogol AD \& Pincus SM. Estrogen and testosterone, but not a non-aromatizable androgen, direct network integration of the hypothalamo-somatotrope (growth hormone)-insulin-like growth factor I axis in the human: evidence from pubertal pathophysiology and sex-steroid hormone replacement. Journal of Clinical Endocrinology and Metabolism 199782 3414-3420.

24 Veldhuis JD, Johnson ML, Veldhuis OL, Straume M \& Pincus S. Impact of pulsatility on the ensemble orderliness (approximate entropy) of neurohormone secretion. American Journal of Physiology 2001281 R1975-R1985.

25 Fisher LD \&van Belle G. Descriptive statistics. In Biostatistics: A Methodology for the Health Sciences, pp 58-74. New York: John Wiley \& Sons, 1996.

26 Hofland LJ, Van Der HJ, Feelders R, van Aken MO, Van Koetsveld PM, Waaijers M, Sprij-Mooij D, Bruns C, Weckbecker G, De Herder WW, Beckers A \& Lamberts SW. The multi-ligand somatostatin analogue SOM230 inhibits ACTH secretion by cultured human corticotroph adenomas via somatostatin receptor type 5. European Journal of Endocrinology 2005152 645-654.

27 Luque RM, Gahete MD, Hochgeschwender U \& Kineman RD. Evidence that endogenous SST inhibits ACTH and ghrelin expression by independent pathways. American Journal of Physiology. Endocrinology and Metabolism 2006291 E395-E403.

28 Silva AP, Schoeffter P, Weckbecker G, Bruns C \& Schmid HA. Regulation of CRH-induced secretion of ACTH and corticosterone by SOM230 in rats. European Journal of Endocrinology 2005153 R7-R10.

29 Thorner MO, Vance ML, Hartman ML, Holl RW, Evans WS, Veldhuis JD, Van Cauter E, Copinschi G \& Bowers CY. Physiological role of somatostatin on growth hormone regulation in humans. Metabolism 199039 40-42.

30 Peterfreund RA \& Vale WW. Ovine corticotropin-releasing factor stimulates somatostatin secretion from cultured brain cells. Endocrinology 1983112 1275-1278.

31 Rigamonti AE, Bonomo SM, Cella SG \& Muller EE. GH and cortisol rebound rise during and following a somatostatin infusion: studies in dogs with the use of a GH-releasing peptide. Journal of Endocrinology $2002 \mathbf{1 7 4} 387-394$. 
32 von Zastrow M \& Castle JD. Protein sorting among two distinct export pathways occurs from the content of maturing exocrine storage granules. Journal of Cell Biology 1987105 2675-2684.

33 Nickel W \& Wieland FT. Biosynthetic protein transport through the early secretory pathway. Histochemistry and Cell Biology 1998 $109477-486$.

34 Bergendahl M, Iranmanesh A, Mulligan T \& Veldhuis JD. Impact of age on cortisol secretory dynamics basally and as driven by nutrient-withdrawal stress. Journal of Clinical Endocrinology and Metabolism 200085 2203-2214.

35 Iranmanesh A, Veldhuis JD, Johnson ML \& Lizarralde G. Twentyfour hour pulsatile and circadian patterns of cortisol secretion in alcoholic men. Journal of Andrology 198910 54-63.
36 Carroll BJ, Cassidy F, Naftolowitz D, Tatham NE, Wilson WH Iranmanesh A, Liu PY \& Veldhuis JD. Pathophysiology of hypercortisolism in depression. Acta Psychiatrica Scandinavica 2007115 90-103.

37 Van Cauter E, Leproult R \& Kupfer DJ. Effects of gender and age on the levels and circadian rhythmicity of plasma cortisol. Journal of Clinical Endocrinology and Metabolism $1996 \mathbf{8 1}$ 2468-2473.

Received 24 July 2008

Accepted 29 July 2008 